\title{
Astragalus alters gut-microbiota composition in type 2 diabetes mice: clues to its pharmacology
}

This article was published in the following Dove Press journal:

Diabetes, Metabolic Syndrome and Obesity: Targets and Therapy

\author{
Xin-Yu Li ${ }^{1,2}$ \\ Liang Shen ${ }^{1,2}$ \\ Hong-Fang $\mathrm{Ji}^{1,2}$ \\ 'Institute of Biomedical Research, \\ Shandong University of Technology, Zibo, \\ Shandong, People's Republic of China; \\ ${ }^{2}$ Shandong Provincial Research Center \\ for Bioinformatic Engineering and \\ Technique, Zibo Key Laboratory of New \\ Drug Development of \\ Neurodegenerative Diseases, School of \\ Life Sciences, Shandong University of \\ Technology, Zibo, Shandong, People's \\ Republic of China
}

Background: Astragalus possesses therapeutic effects for type 2 diabetes (T2D), while its action mechanisms remain to be elucidated. In view of the pathogenic associations between gut microbiota and T2D, we explored the effect of astragalus on gut-microbiota composition of T2D mice. Materials and methods: Modulation effects of astragalus on gut microbiota of T2D-model mice were assessed by 16S rRNA gene sequencing.

Results: Inhibited blood-glucose and body-weight levels of T2D mice by astragalus were accompanied by gut microbiota-composition alteration. Astragalus administration significantly increased gut-microbiota richness and diversity in T2D mice and significantly altered the abundance of several bacterial taxa, inducing increased abundance of Lactobacillus and Bifidobacterium. PICRUSt software revealed the relationship between astragalus and T2D.

Conclusion: Due to previously reported decreased gut-microbiota richness and diversity and reduced abundance of key species of Lactobacillus and Bifidobacterium, more studies are encouraged to explore the contribution of gut-microbiota alteration by astragalus to its anti-T2D effect.

Keywords: astragalus, type 2 diabetes, gut microbiota, alteration, 16S rRNA gene sequencing

\section{Introduction}

Type 2 diabetes (T2D) is a chronic metabolic disease caused by the interaction of inherited and environmental factors. It was estimated that almost 425 million adults worldwide suffered from diabetes in 2017, and this number is projected to increase to 628 million by $2045 .{ }^{1}$ The steadily increasing number of people living with T2D has created a global economic burden. ${ }^{2}$ In addition, people with T2D are prone to developing severe complications, such as cardiovascular disease, diabetic nephropathy, diabetic neuropathy, and diabetic retinopathy. ${ }^{3-6}$ Therefore, there is an urgent need for prevention and early intervention of T2D.

Astragalus has been used in traditional Chinese medicines for thousands of years for its pharmacological effects. In the past decade, numerous studies on human and animal models have shown that astragalus has an antidiabetic effect. ${ }^{7-9}$ Owing to the poor bioavailability of the main active components of astragalus, such as saponins and flavonoids, more effort is needed to explore its underlying action mechanisms. ${ }^{10-12}$

In recent years, many animal and humans studies have suggested that gut microbiota may play an etiological role in $\mathrm{T} 2 \mathrm{D},{ }^{13-16}$ and gut microbiota have been proposed to be potential therapeutic targets of this disease. As such, it is rational to hypothesize that astragalus may exert anti-T2D effects through altering the composition of gut microbiota. This stimulated us to explore the effect of oral administration of astragalus on gut microbiota of T2D-model mice by $16 \mathrm{~S}$ rRNA
Correspondence: Liang Shen Institute of Biomedical Research, Shandong University of Technology, Zibo, Shandong, People's Republic of China

Tel +865332782220

Email shen@sdut.edu.cn 
gene sequencing, providing clues to understand the mechanism of action of this natural agent.

\section{Materials and methods}

Astragalus in brown-yellow fine-powder form containing $70 \%$ astragalan and $10 \%$ total saponins, was purchased from Huayue Chemical Products (Henan, China). Carboxymethylcellulose sodium (CMC-Na) was obtained from Sigma-Aldrich (St Louis, MO, USA). Accu-Chek was purchased from Roche Diagnostics (Mannheim, Germany). BKS.Cg-Dock7m +/+ Leprdb/Nju mice (5 weeks old) were purchased from the Model Animal Research Center of Nanjing University (Nanjing, China).

Mice were housed one per cage in a specific pathogenfree animal lab and maintained under standard conditions: a 12-hour light/dark cycle with room temperature at $22^{\circ} \mathrm{C}$ $\pm 2^{\circ} \mathrm{C}$ and $50 \% \pm 5 \%$ humidity, and with ad libitum access to food and water. Ten mice were acclimatized to the laboratory environment for 1 week before the experiment. All mice were divided into two equal groups randomly: a control group and an astragalus-administered group. The astragalus-administered group received astragalus dissolved in $0.5 \%$ CMC-Na by gavage at a dosage of $1 \mathrm{~g} / \mathrm{kg}$ body weight per day, with mice fed a standard diet with $0.5 \%$ CMC-Na buffer as the control group. Mice were treated for 15 days once daily. Animal experiments were approved by the Animal Use Subcommittee of the Shandong University of Technology. Our use of experimental animals was in compliance with the Guide for the Care and Use of Laboratory Animals. Blood samples were withdrawn from an orbit vein after 12 hours' fasting. Blood was centrifuged at 3,000 rpm for 10 minutes to obtain plasma. Fasting blood glucose (FBG) was measured with the Accu-Chek according to the manufacturer's instructions.

Fresh mice feces were collected into individual sterile Ependorf tubes and then frozen immediately at $-80^{\circ} \mathrm{C}$ until DNA extraction. DNA extraction from each fecal sample was conducted by phenol trichloromethane methods. The extracted DNA concentration was determined by NanoDrop (Thermo Fisher Scientific). After DNA extraction from the feces samples, we used PCR amplification and pyrosequenced the $\mathrm{V}_{3}$ and $\mathrm{V}_{4}$ regions of the bacterial $16 \mathrm{~S}$ ribosomal RNA gene. Amplicon-sequencing libraries were sequenced using the Illumina Miseq platform for paired-end reads of $300 \mathrm{bp}$. Several $\alpha$-diversity indices were analyzed to evaluate the effect of astragalus on gutmicrobiota richness and diversity of T2D mice. Dominant bacterial community differences between groups were detected employing linear discriminant analysis combined with effect size measurements (LEfSe). LEfSe was used to identify species most characteristic of different sample types. LEfSe results were visualized using taxonomy barchart and cladogram plots, as implemented on the LEfSe website (http://huttenhower.sph.harvard.edu/galaxy). Microbial functions were predicted with PICRUSt software. ${ }^{17}$ Relevant predicted genes and their functions were aligned with the Kyoto Encyclopedia of Gene and Genomes (KEGG) database and differences among groups compared with STAMP software.

FBG and body-weight parameters were analyzed with SPSS 16.0. Data comparisons among different groups were analyzed by ANOVA. Graphic presentations were achieved with GraphPad Prism 6 (GraphPad Software, San Diego, IL, USA).

\section{Results}

\section{Effects on FBG and body-weight levels}

The effect of astragalus administration on FBG and bodyweight levels was evaluated through comparison with the control group. The results showed that astragalus administration for 15 days reduced FBG and body weight, as shown in Figure 1.

\section{Overall structural alteration of gut microbiota}

All fecal samples were examined using Illumina highthroughput sequencing. A data set consisting of 462,040 total sequence-read counts was generated, and the average number of sequences obtained was 46,204 for each sample. Altogether, 433 operational taxonomic units were exhibited at a $97 \%$ similarity level. According to the Venn diagram in Figure 2A, there were 247 shared operational taxonomic units between the two groups, with eleven unique to the control group and 175 to the astragalusadministered group. Rarefaction curves plateaued with the current sequencing, indicating that most gut microbial organisms in each sample were captured with the current sequencing depth (Figure 2B).

To explore the effect of astragalus on the richness and diversity of gut microbiota, we analyzed the $\alpha$-diversity metrics (including Chao1, PD_whole_tree, Shannon, and Simpson) of the control and astragalus-administered groups. It was found that astragalus administration significantly increase gut-microbiota diversity and diversity of T2D mice (see Table 1). 
A

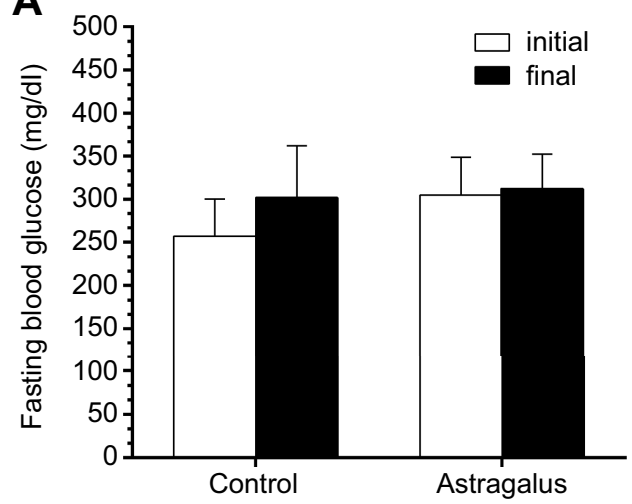

B

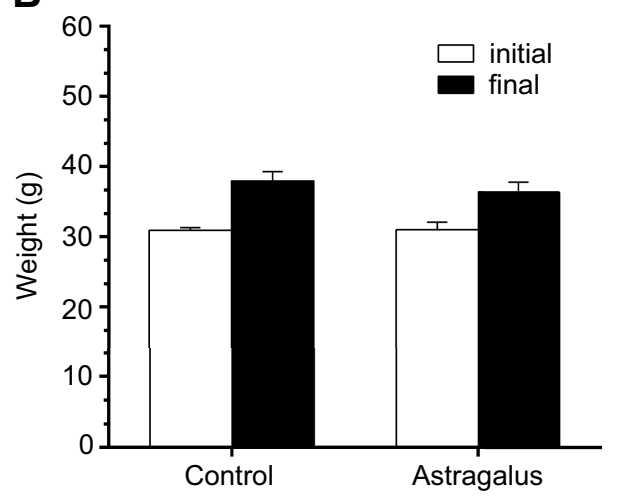

Figure I Effect of astragalus on fasting blood-glucose $(\mathbf{A})$ and body-weight $(\mathbf{B})$ levels in type 2 diabetes mice. Values represent mean $\pm \mathrm{SE}(\mathrm{n}=5)$.

A

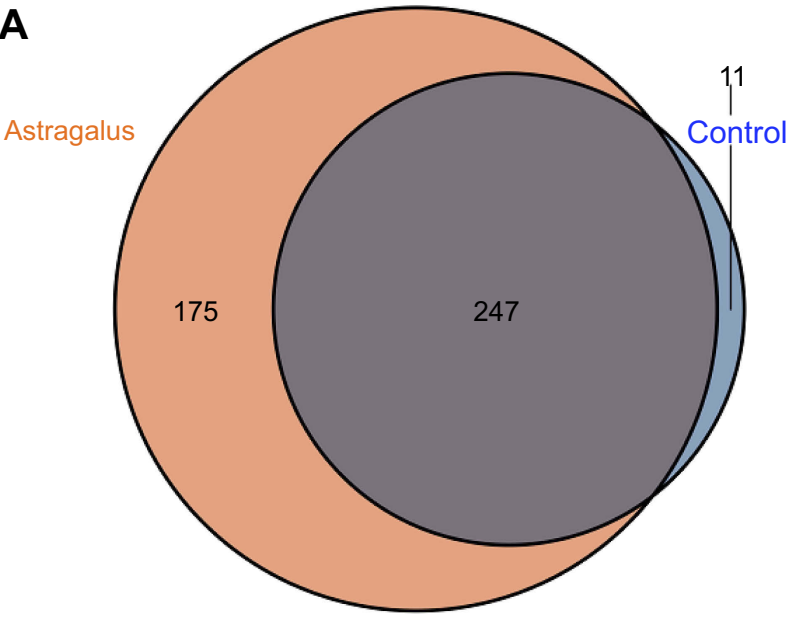

B

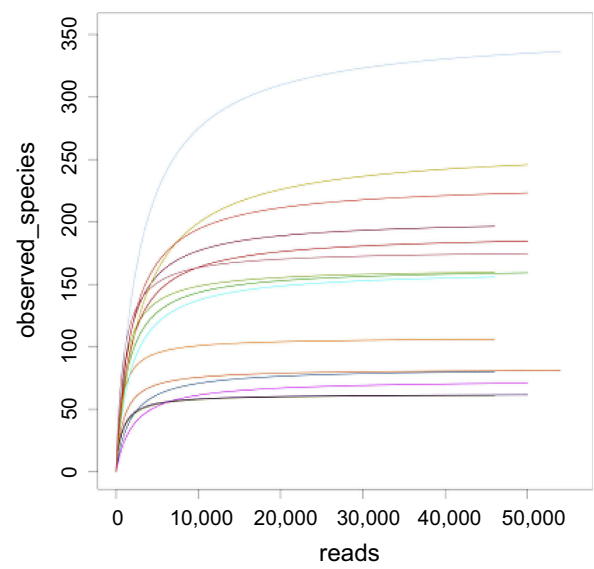

Figure 2 Evaluation of Illumina MiSeq data showing that astragalus altered the overall composition of gut microbiota in type 2 diabetes mice.

Notes: (A) Venn diagram of shared operational taxonomic units in astragalus-administered and control groups; (B) rarefaction curves determined at the $97 \%$ similarity level.

Table I Gut-microbiota diversity and richness indices of control and astragalus-administered groups

\begin{tabular}{|l|l|l|l|l|}
\hline & Chaol & PD_whole_tree & Shannon & Simpson \\
\hline Control & 142.0 & 9.8499 & 4.3877 & 0.9013 \\
Astragalus & 272.3 & 19.1878 & 5.4599 & 0.9560 \\
$\boldsymbol{P}$ & 0.0317 & 0.0317 & 0.0079 & 0.0556 \\
\hline
\end{tabular}

\section{Bacterial composition analysis}

Bacterial composition in the astragalus-administered group and control group was then compared. As illustrated in Figure 3, A and B), Bacteroidetes, Firmicutes and Proteobacteria were the three dominant phyla in all samples. The 20 most abundant genera in the two groups are shown in Figure 3C. A total of three genera exhibited significant differences in abundance between the astragalus-administered group and the control group (Figure 3D). Further analysis found that the relative abundance of Oscillibacter significantly increased from $0.13 \%$ to $1.05 \%(P<0.01)$ after administration of astragalus.

\section{Taxonomic analysis}

LEfSe was used to explore significant changes and relative richness in the bacterial community in the control and astragalus groups (Figure 4). LEfSe results were visualized using taxonomy bar-chart and cladogram plots. Three phylum (Firmicutes, Acidobacteria, and Gemmatimonadetes) were enriched in the astragalus- 

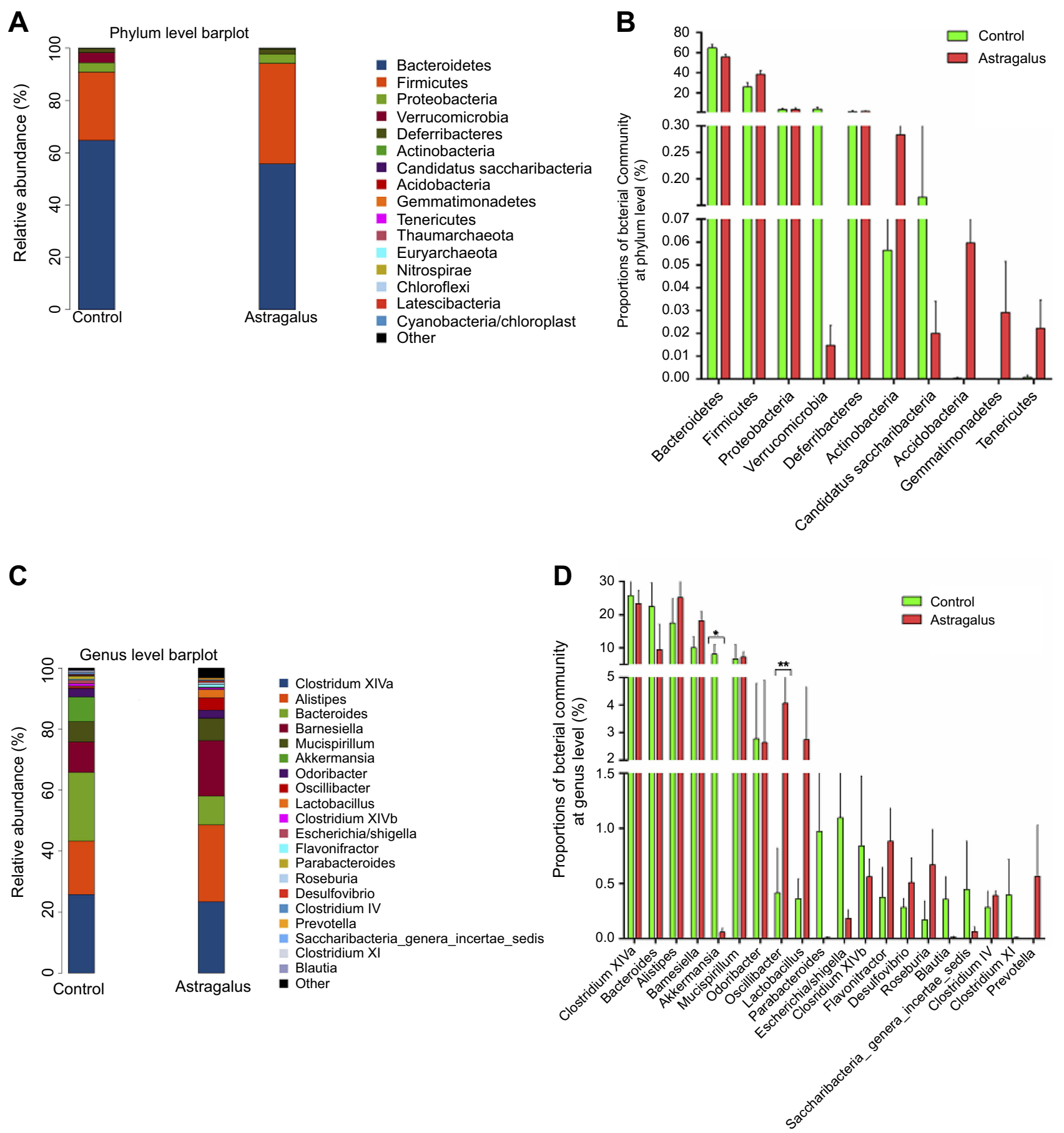

Figure 3 Comparisons of bacterial community abundance between the control and astragalus- administered groups.

Notes: (A) Abundance bar plot at phylum level; (B) significance of top ten bacterial community abundance at phylum level; (C) abundance bar plot at genus level. (D) significance of top 20 bacterial community abundance at genus level. $* P<0.05 ; * * P<0.01$.

adminisered group and none in the control group. One genus and six genera were enriched in the control and astragalus-administered group, respectively. Astragalus administration significantly inhibited the growth of Clostridium cluster XI, and increased the growth of Lactobacillus and Bifidobacterium in T2D mice.

\section{Metabolic function analysis}

PICRUSt analysis was used to predict the metabolic functions of gut microbiota influenced by astragalus in $\mathrm{T} 2 \mathrm{D}$ mice. The results revealed that 13 and 31 KEGG pathways were changed in the astragalus group at levels 2 (Figure 5A) and 3 (Figure 5B), respectively, 


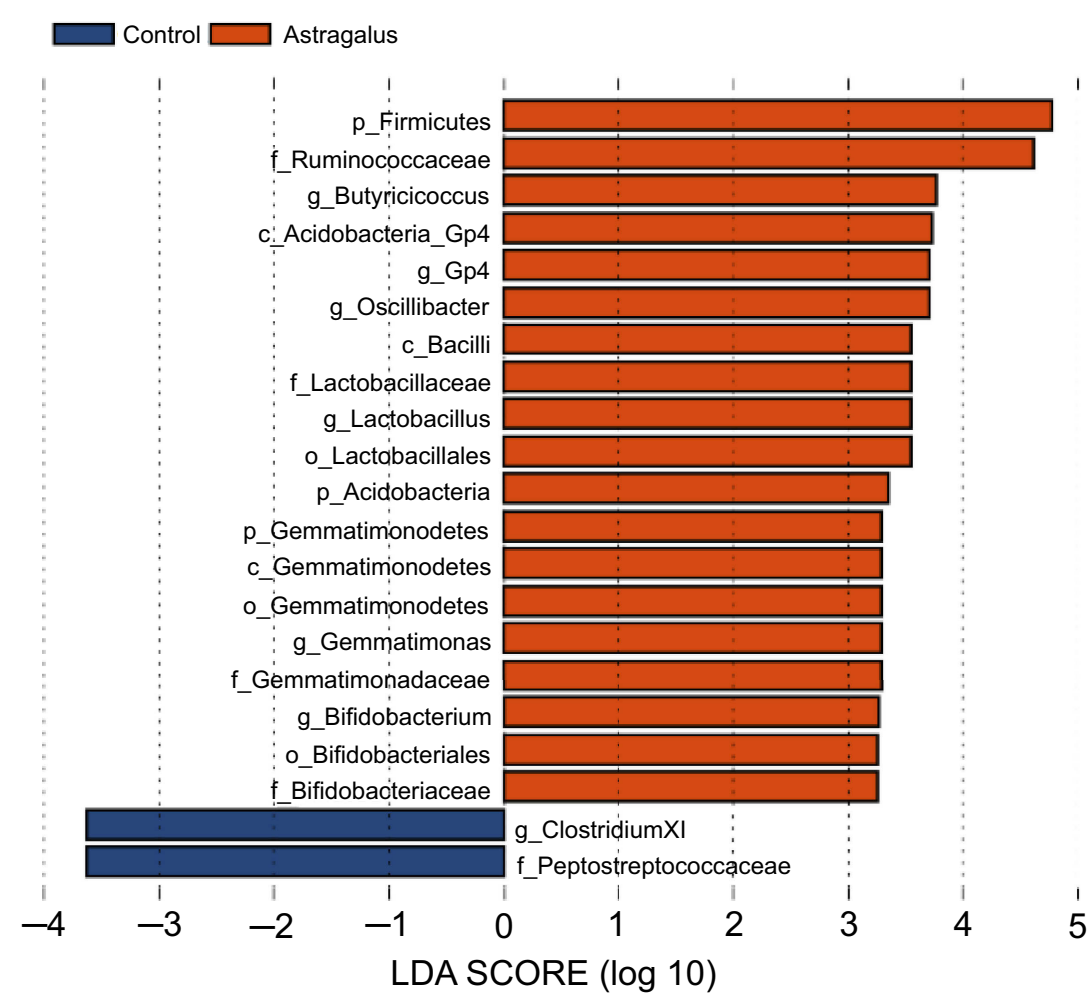

Figure 4 Linear discriminant analysis (LDA) combined witheffect-size measurements at all levels of control and astragalus-administered groups.

Notes: LDA scores that were greater in astragalus males shown in red, whereas the one (Clostridium XI) LDA score that was elevated in control males is depicted in blue. The LDA score of Lactobacillus and Bifidobacterium were greater in astragalus males than control males. Only LDA scores $\geq 2$ are listed.

among which six were increased and seven decreased in comparison with the control group at level 2.

In particular, we found several interesting changes wherein 31 KEGG pathways at level 3 had changed. Firstly, the biosynthesis processes of bacteria, such as nucleotide metabolism, enzyme families, translation, cell growth, death, replication, repair, and motility (level 2) and nucleotide metabolism, including that of pyrimidine and cytoskeleton proteins (level 3), had increased in the astragalus group in comparison with the control group. In addition, the metagenome of the control group had been enriched in pathways related to xenobiotic biodegradation and metabolism, including styrene degradation, aminobenzoate degradation, caprolactam degradation, metabolism of xenobiotics by cytochrome P450, drug metabolism with cytochrome P450, and chlorocyclohexane and chlorobenzene degradation; lipid metabolism, including arachidonic-acid metabolism and fatty-acid metabolism; and animo-acid metabolism, including phenylalanine metabolism, tyrosine metabolism, lysine degradation, valine, leucine, and isoleucine degradation, and tryptophan metabolism.

\section{Discussion}

In recent years, many traditional Chinese medicines have been tried to treat $\mathrm{T} 2 \mathrm{D},{ }^{18-20}$ among which astragalus is considered a promising antidiabetic natural agent, but its mechanism of action needs to be explored. Inspired by the recent findings of gut-microbiota regulation in interpreting the pharmacology of anti-T2D agents, ${ }^{21}$ the present work studied alterations ingut microbiota of T2D mice through astragalus administration. It was found that astragalus prevented increases of FBG levels and body weight. Characterization of gut microbiota showed that astragalus administration significantly increased microbial diversity and richness and altered the relative abundance of several key bacterial species.

It has been reported that gut-microbiota diversity and richness decrease in diabetic mice compared to controls ${ }^{22}$ The "normalization" effect of astragalus observed in the current study may make an important contribution to its pharmacological effect. In addition, both human and animal studies have indicated that abundance of Lactobacillus and Bifidobacterium decreases in diabetic rats and T2D patients. ${ }^{23,24}$ Djurasevic et al found that virgin coconut oil 


\section{$\square$ Control $\square$ Astragalus}

A

Xenobiotics_biodegradation_and_metabolism

Infectious_diseases :

Nucleotide_metabolism

Enzyme_families $\equiv$

Translation

Celluiar_processes_and_signaling

Excretory_system |

Cell_growth_and_death $\mathrm{G}$

Replicaton_and_repair

Poorly_characterized

Cell_motility

Carbohydrate_metabolism

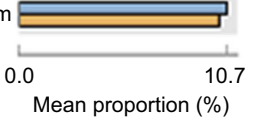

$\square$ Control $\square$ Astragalus

\section{B}

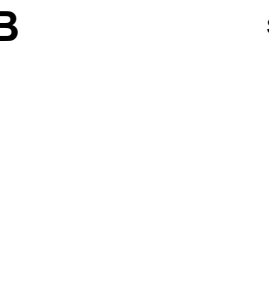

Selenocompound_metabolism $\boxminus$

Styrene_degradation |

Aminobenzoate_degradation日

Peptidoglycan_biosynthesis

Geraniol_degradation

Caprolactam_degradation \$
Function_unknown

Glycosyltransferases $\square$

Metabolism_of_xenobiotics_by_cytochrome_P450 |

Inorganic_ion_transport_and_metabolism

Retinol_metabolism |

Transcription_related_proteins |

Drug_metabolism_cytochrome_P450 |

Lysine_biosynthesis

Phenylalanine_metabolism 日

Pyrimidine_metabolism

Tyrosine_metabolism

Penicillin_and_cephalosporin_biosynthesis |

Arachidonic_acid_metabolism |

Chromosome

Chlorocyclohexane_and_chlorobenzene_degradation |

Bacterial_secretion_system

Lysine_degradation 8

Cell_cycle - caulobacter

Metabolism_of_cofactors_and_vitamins ?

Glutathione_metabolism 日

Fatty_acid_metabolism 日

Valine,_leucine_and_isoleucine_degradation $日$

Tryptophan_metabolism 日

Cytoskeleton_proteins

Biosynthesis_and_biodegradation_of_secondary_metab...

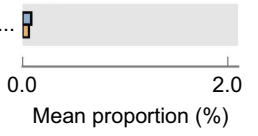

$95 \%$ confidence intervals

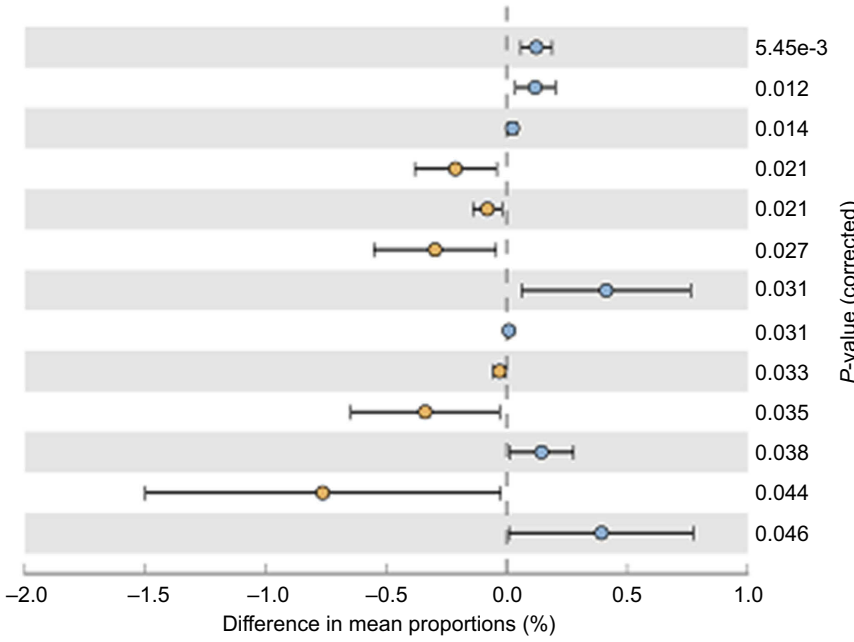

$5.45 \mathrm{e}-3$

0.014

0.021

$\frac{1}{1}$

0.044

Difference in mean proportions (\%)

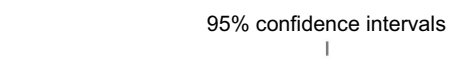


affected some secondary parameters in diabetic rats and significantly increased the abundance of probiotic bacteria, such as Lactobacillus, Allobaculum, and Bifidobacterium spp. ${ }^{25}$ Previous studies also found increased relative abundance of Bifidobacterium in T2D patients after treatment with metformin. ${ }^{26,27}$ Although no metabolic pathway associated with differential bacteria was found in functional prediction analysis, the biosynthesis processes of bacteria was increased in the astragalus group compared to the control group. This suggested that the addition of astragalus promoted cellular processes, but the current findings need to be further verified.

To summarize, the present findings indicated that inhibition of FBG and body-weight levels in T2D mice was associated with alterations in gut-microbiota composition. Increased gut-microbiota diversity and richness and regulation of key bacterial species abundance may be involved in the antidiabetic effect of astragalus. Further studies are needed to evaluate the contribution of gut-microbiota alteration to the anti-T2D activity of astragalus, which is important to understand the pharmacology of this agent better.

\section{Abbreviation list}

T2D, type 2 diabetes; $\mathrm{CMC}-\mathrm{Na}$, carboxymethylcellulose sodium.

\section{Acknowledgments}

This work was supported by the Shandong Provincial Natural Science Foundation (grant ZR2018MH010), Shandong Provincial Key Research and Development Program (grant 2018GSF121001), and the Talent Program of Zibo.

\section{Disclosure}

The authors report no conflicts of interest in this work.

\section{References}

1. International Diabetes Federation. IDF Diabetes Atlas. 8th ed. Brussels: IDF; 2017.

2. Bommer C, Sagalova V, Heesemann E, et al. Global economic burden of diabetes in adults: projections from 2015 to 2030. Diabetes Care. 2018;41:963-970. doi:10.2337/dc17-1962

3. Beckman JA, Creager MA, Libby P. Diabetes and atherosclerosis: epidemiology, pathophysiology, and management. JAMA. 2002;287:2570-2581.

4. Chen L, Magliano DJ, Zimmet PZ. The worldwide epidemiology of type 2 diabetes mellitus-present and future perspectives. Nat Rev Endocrinol. 2011;8:228-236. doi:10.1038/nrendo.2011.183
5. Kraemer FB, Ginsberg HN, Gerald M, Reaven M. Demonstration of the central role of insulin resistance in type 2 diabetes and cardiovascular disease. Diabetes Care. 2014;37:1178-1181. doi:10.2337/ de13-2668

6. Hoogwerf BJ. Complications of diabetes mellitus. Int J Diabetes Dev Ctries. 2005;25:63-69. doi:10.4103/0973-3930.22774

7. Lau KM, Lai KK, Liu CL. Synergistic interaction between astragali radix and rehmanniae radix in a chinese herbal formula to promote diabetic wound healing. J Ethnopharmacol. 2012;141:250-256. doi:10.1016/j.jep.2012.02.025

8. Zhang K, Pugliese M, Pugliese A, Passantino A. Biological active ingredients of traditional Chinese herb astragalus membranaceus on treatment of diabetes: a systematic review. Mini Rev Med Chem. 2015;15:315-329. doi:10.2174/13895575156661502 27113431

9. Wang Y, Lin C, Ren Q, Liu Y, Yang X. Astragaloside effect on TGF$\beta 1$, SMAD2/3, and $\alpha$-SMA expression in the kidney tissues of diabetic KKAy mice. Int J Clin Exp Pathol. 2015;8:6828.

10. Yu K, Chen F, Li C. Absorption, disposition, and pharmacokinetics of saponins from Chinese medicinal herbs: what do we know and what do we need to know more? Curr Drug Metab. 2012;13:577-598. doi:10.2174/1389200211209050577

11. Manach C, Williamson G, Morand C, Scalbert A, Rémésy C. Bioavailability and bioefficacy of polyphenols in humans. I. Review of 97 bioavailability studies. Am $J$ Clin Nutr. 2005;81:230S-242S.

12. Shen L, Ji HF. Intestinal microbiota and metabolic diseases: pharmacological Implications. Trends Pharmacol Sci. 2016;37:169-171. doi:10.1016/j.tips.2015.11.010

13. Barlow GM, Yu A, Mathur R. Role of the gut microbiome in obesity and diabetes mellitus. Nutr Clin Pract. 2015;30:787-797. doi: $10.1177 / 0884533615609896$

14. Sohail MU, Althani A, Anwar H, Rizzi R, Marei HE. Role of the gastrointestinal tract microbiome in the pathophysiology of diabetes mellitus. J Diabetes Res. 2017;2017:9631435. doi:10.1155/2017/ 9631435

15. Brunkwall L, Orho-Melander M. The gut microbiome as a target for prevention and treatment of hyperglycaemia in type 2 diabetes: from current human evidence to future possibilities. Diabetologia. 2017;60:943-951. doi:10.1007/s00125-017-4278-3

16. Karlsson FH, Tremaroli V, Nookaew I, et al. Gut metagenome in European women with normal, impaired and diabetic glucose control. Nature. 2013;498:99-103. doi:10.1038/nature12184

17. Kanehisa M, Goto S, Sato Y, Furumichi M, Tanabe M. KEGG for integration and interpretation of large-scale molecular data sets. Nucleic Acids Res. 2012;40:109-114.

18. Li W, Zheng H, Bukuru J, De Kimpe N. Natural medicines used in the traditional Chinese medical system for therapy of diabetes mellitus. J Ethnopharmacol. 2004;92:1-21. doi:10.1016/j.jep.2003. 12.031

19. Hsu PC, Tsai YT, Lai JN, Wu CT, Lin SK, Huang CY. Integrating traditional Chinese medicine healthcare into diabetes care by reducing the risk of developing kidney failure among type 2 diabetic patients: a population-based case control study. J Ethnopharmacol. 2014;156:358-364. doi:10.1016/j.jep.2014.08.029

20. Poon TY, Ong KL, Cheung BM. Review of the effects of the traditional Chinese medicine rehmannia six formula on diabetes mellitus and its complications. J Diabetes. 2011;3:184-200. doi:10.1111/ j.1753-0407.2011.00130.x

21. Wu H, Esteve E, Tremaroli V, et al. Metformin alters the gut microbiome of individuals with treatment-naive type 2 diabetes, contributing to the therapeutic effects of the drug. Nat Med. 2017;23:850-858. doi:10.1038/nm.4265 
22. Zhang Q, Yu H, Xiao X, Hu L, Xin F, Yu X. Inulin-type fructan improves diabetic phenotype and gut microbiota profiles in rats. PeerJ. 2018;6:e4446. doi:10.7717/peerj.4446

23. Yan X, Feng B, Li P, Tang Z, Wang L. Microflora disturbance during progression of glucose intolerance and effect of sitagliptin: an animal study. J Diabetes Res. 2016;2016:2093171. doi:10.1155/2016/ 2093171

24. Barengolts E, Green SJ, Eisenberg Y, et al. Gut microbiota varies by opioid use, circulating leptin and oxytocin in African American men with diabetes and high burden of chronic disease. PLoS One. 2018;13:e0194171. doi:10.1371/journal.pone.0194171
25. Djurasevic S, Bojic S, Nikolic B, et al. Beneficial effect of virgin coconut oil on alloxan-induced diabetes and microbiota composition in rats. Plant Foods Hum Nutr. 2018;73:295-301. doi:10.1007/s11130-018-0689-7

26. Forslund K, Hildebrand F, Nielsen T, et al. Disentangling type 2 diabetes and metformin treatment signatures in the human gut microbiota. Nature. 2015;528:262-266. doi:10.1038/nature15766

27. de la Cuesta-Zuluaga J, Mueller NT, Corrales-Agudelo V, et al. Metformin is associated with higher relative abundance of mucindegrading akkermansia muciniphila and several short-chain fatty acid-producing microbiota in the gut. Diabetes Care. 2017;40:5462. doi:10.2337/dc16-1324

\section{Publish your work in this journal}

Diabetes, Metabolic Syndrome and Obesity: Targets and Therapy is an international, peer-reviewed open-access journal committed to the rapid publication of the latest laboratory and clinical findings in the fields of diabetes, metabolic syndrome and obesity research. Original research, review, case reports, hypothesis formation, expert opinion and commentaries are all considered for publication. The manuscript management system is completely online and includes a very quick and fair peer-review system, which is all easy to use. Visit http://www.dovepress.com/testimonials.php to read real quotes from published authors.

Submit your manuscript here: https:/www.dovepress.com/diabetes-metabolic-syndrome-and-obesity-targets-and-therapy-journal 